\title{
Investigating complementary computational and empirical activities for students learning diffusion
}

\author{
Daniel P. Weller, ${ }^{1}$ Kathleen Hinko, ${ }^{2}$ and Vashti Sawtelle ${ }^{2}$ \\ ${ }^{1}$ Department of Chemical Engineering and Materials Science \\ ${ }^{2}$ Lyman Briggs College and Department of Physics and Astronomy \\ Michigan State University, 220 Trowbridge Rd., East Lansing, MI, USA, 48824
}

\begin{abstract}
There has been a push in physics education to incorporate computation into the classroom in a meaningful way; however, there is still much to learn about how combining computational work with traditional laboratory work can support student learning. This scenario is especially relevant to a studio classroom where lecture and lab activities are integrated. For an introductory physics for the life sciences studio course, we have developed an empirical microscope lab to complement a computational activity that models the diffusive motion of particles in solution. The microscope lab was designed for students to develop and test their own hypotheses about microbead solutions experiencing diffusion, while the computational exercise served as a model for visualizing diffusion on the microscopic level. Taking a resource theory perspective, we compare the empirical and computational activities by analyzing student responses to pre-lab, post-lab, and final exam questions. We find that students perceive the two in-class activities as "realistic" or "idealized," and we present affordances and constraints of studying diffusion by computational and empirical means.
\end{abstract}

\section{INTRODUCTION}

In physics education, there has been a movement to transition large lecture introductory physics courses to a studiostyle environment that integrates laboratory activities with lecture and problem-solving [1, 2]. In parallel with these curricular reforms, incorporating computation into the undergraduate curriculum has been a large priority [3]. As these teaching strategies become more commonplace in university classrooms, it is important to understand the affordances and constraints of computational simulations as well as empirical investigations. The aim of this work is to explore how computation and experimentation can complement each other to support student learning in introductory physics.

Our investigation is situated in a calculus-based Introductory Physics for the Life Sciences (IPLS) first semester course. The class consisted of 156 students (4 sections) over one semester, taught by two different instructors, with most of the students being life sciences and pre-med majors. The class utilized a studio model with a particular emphasis on cross-cutting concepts including diffusion, chemical energy, and Gibbs free energy [1, 4]. In particular, we focused on diffusion as a rich interdisciplinary topic that students could investigate by both computational and experimental means throughout the mechanics-focused semester.

In this paper, we will present analysis of student learning in paired computational and empirical activities on diffusion. This innovative work will (1) unpack how students see these activities as representing "real" and "ideal" models; (2) explore the impacts of combining empirical investigations with computational simulations; and (3) investigate students' learning of diffusion from a physics perspective.

\section{IN-CLASS ACTIVITIES}

An objective of this work was studying how student learning was affected by two complementary approaches for in- vestigating diffusion. In the fifth week of the course, students began the unit on diffusion with a computational activity that simulates the random motion of differently sized spheres. Previously, Tunstall et al. analyzed homework responses and identified conceptual resources that students activated when defining diffusion in this context [5]. The study reported herein examines the benefit of adding an empirical microscope lab to the diffusion sequence, administered the week after the computational activity. The computational activity was developed in the year prior to the microscope lab, and the two activities were meant to go hand-in-hand. Figure 1 displays a course timeline detailing activities and assignments pertaining to diffusion.

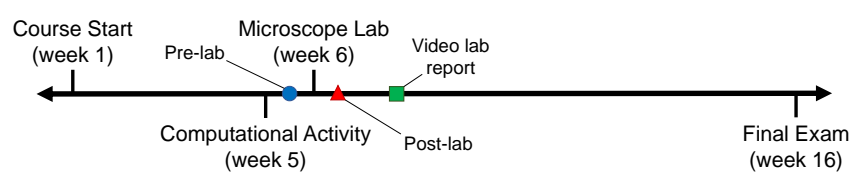

FIG. 1. Course timeline for Physics 1 for the life sciences (LB273) with symbols indicating due dates for pre-lab (week 6), post-lab (week 6), and video lab report (week 7) assignments.

\section{A. Computational activity modeling diffusion}

To begin the unit on diffusion, students computationally simulated the motion of several large ("solute") spheres in a box with many smaller ("solvent") spheres. They edited a minimally working code through the use of VPython and Glowscript (free online Python compiler). The program initially assigns the particles a random velocity, and then velocities are calculated and updated iteratively. After properly coding for momentum conservation between the colliding objects, students obtain a visual display like that shown in Fig. 2a. From there, they can explore numerous aspects of the code to change visual features, modify computational parameters, and test relationships between different variables. For example, the solvent molecules can be made transparent with 
a motion trail added to the solute particles as displayed in Fig. 2b. Alternatively, students can change the object masses or number of objects to examine how momentum conservation or concentration affects diffusion. The goal of this activity is for students to gain a collision-based visual model of diffusion, while considering the effects of different variables on the diffusive process.

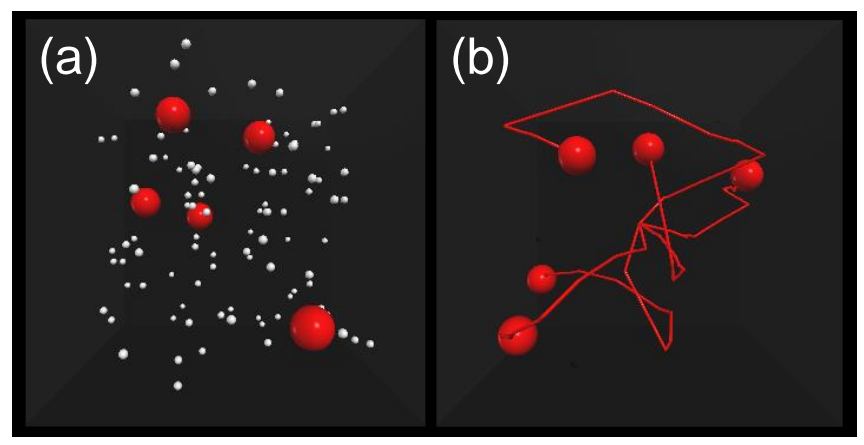

FIG. 2. Display output of the computational activity modeling diffusion (a) with conservation of momentum correctly coded and (b) with visual features and computational parameters modified.

\section{B. Empirical investigation of diffusion with microscopes}

For the experimental hands-on lab activity, students were asked to view microbead solutions under a microscope and test their own hypotheses about diffusion in these solutions. The empirical microscope lab was adapted from Moore et al. [6]. It was designed to be open-ended such that students could form their own ideas, instead of following a traditional guided laboratory exercise. Prior to running their own experiments, students familiarized themselves with the equipment by focusing, viewing, and capturing images of silica beads in a water solution (see Fig. 3). Cameras attached to the microscope (via Amscope software) were used to capture picture and video data from the microscope viewing window. Then, students completed a pre-lab assignment and designed their own experiment to execute in the next class period.

For students to be able to empirically experience diffusion and design an experiment with a range of measurable variables, we needed to provide them with samples that would meet these requirements. Thus, solutions of silica or polystyrene microbeads of varying sizes $(1,2,5 \mu \mathrm{m})$ and solvents (water and $25 \%$ glycerol) were provided. Students were tasked with hypothesizing how a variable (e.g. bead size or solvent) would affect the diffusion rate and designing an experiment to test it.

Many different solutions were provided to allow students to investigate a multitude of different experimental variables. For instance, some students looked into the relationship between bead size and displacement over time, while others studied the effect of solvent and viscosity on the motion of the beads. Moreover, students were encouraged to think creatively about their experiments; thus, other groups investi-

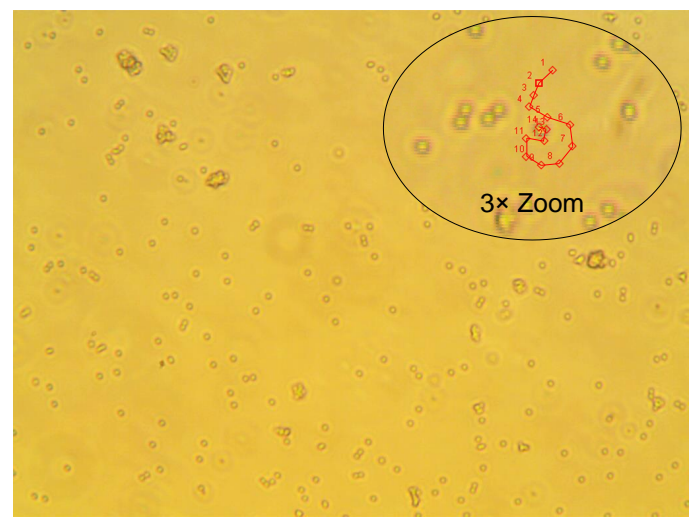

FIG. 3. Microscope image ( $40 \times$ magnification) of $2 \mu \mathrm{m}$ silica beads in water with the inset displaying position points (obtained via Tracker software) for one microbead over several frames.

gated the effects of temperature, concentration, and barriers on bead motion.

After collecting data in class, students used Tracker software to obtain numerical information from their recorded videos and completed a post-lab assignment. Lastly, students presented their final results as a video lab report [7].

\section{METHODOLOGY}

To investigate how the addition of the empirical lab influenced students' ideas about diffusion, we gathered data from the microscope pre-lab and post-lab assignments, as well as final exams. Students were assigned pre-lab and post-lab questions to target their understanding of physics concepts and experimental factors (e.g., tilt in the table, mixing of bead solutions, other outside influences). Specifically, our analysis focused on how students compared and contrasted the two activities before and after completing the microscope lab. Below are the parallel pre/post-lab questions that we examined in our analysis.

Pre-lab: Based on what you saw in the computational activity, what do you expect the motion of the beads in the microscope will look like? Consider what might look similar or different between the two activities.

Post-lab: Compare/contrast the motion of the particles in your videos to the motion of the particles in the " $3 D$ Diffusion” simulation.

Student responses to the above questions were initially coded by looking for indicator phrases that matched the resources identified by Tunstall et al. [5]. These indicator phrases include discussion of concentration gradients, collisions, and spreading of energy. Subsequently, several additional prevalent ideas emerged in our analysis of student responses. Hence, we coded for mentions of physics concepts such as gravity, friction, and particle mass/size; and experi- 
TABLE I. Codebook for student responses pertaining to the realistic or idealistic aspects of the computational activity and empirical lab.

\begin{tabular}{|c|c|c|}
\hline Code & Indicator Phrase & Example \\
\hline \multirow{5}{*}{$\begin{array}{l}\text { Realistic } \\
\quad \text { or } \\
\text { Idealistic }\end{array}$} & $\begin{array}{l}\text { Real, reality, real life, actual, actuality, ac- } \\
\text { tual life }\end{array}$ & $\begin{array}{l}\text { "I think the microscope lab will be different because computer generated things } \\
\text { are different than real, physical things. In real life, the beads will stop moving by } \\
\text { themselves, eventually." }\end{array}$ \\
\hline & Fake & $\begin{array}{l}\text { "The main difference will be that the beads won't look like fake computer- } \\
\text { simulated spheres." }\end{array}$ \\
\hline & $\begin{array}{l}\text { Perfect, theoretical, ideal, idealized, con- } \\
\text { trolled, imperfect, not ideal }\end{array}$ & $\begin{array}{l}\text { "The computational activity we did had theoretical and ideal conditions while the } \\
\text { microscope experiment is not ideal." }\end{array}$ \\
\hline & $\begin{array}{l}\text { Other factors, didn't account for the effect of } \\
\text { other variables }\end{array}$ & $\begin{array}{l}\text { "There will also be other natural factors that weren't seen in the computational } \\
\text { model that we'll see in the experiment (i.e. gravity)." }\end{array}$ \\
\hline & $\begin{array}{l}\text { Accurate representation, better representa- } \\
\text { tion, poor representation }\end{array}$ & $\begin{array}{l}\text { "The simulation poorly represents the motion of the silica beads, but perhaps } \\
\text { represents the motion of the solvent particles better." }\end{array}$ \\
\hline
\end{tabular}

mental factors including the beads settling over time, the concentration of particles in solution, and systematic or random error. These codes were determined through multiple iterations of finding prevalent indicator phrases and then re-coding the same selection to refine the categorization scheme.

Additionally, we attempted to identify affordances and constraints from student responses to pre/post-lab questions. However, the affordances and constraints of the empirical and computational activities were difficult to consistently code from those responses because students did not always make a value statement when identifying features of the two different activities. Therefore, a set of final exam questions was given to students explicitly asking them to describe one advantage and one limitation of modeling diffusion with a computational activity and observing diffusion with a physical lab activity. Slightly different versions of the final exam questions were asked to encourage a diversity of responses focusing on affordances and constraints. Responses to final exam questions were coded in the same manner as pre-lab and postlab responses (described in the previous paragraph).

\section{FINDINGS FROM STUDENT RESPONSES}

Responses to pre/post-lab and final exam questions were coded emergently. A prevalent theme emerged from student responses: the "realistic" or "idealized" aspects of the two activities. We also present the affordances and constraints that students identified in final exam responses.

\section{A. Realistic and idealistic themes}

In the analysis of pre/post-lab data, the realistic and idealistic aspects of the activities emerged as a common theme in student responses. We use the realistic code to describe when students used indicator phrases related to reality, real life, what would actually happen, accounting for other factors, being an accurate representation, etc. Whereas the idealistic code refers to students using indicator phrases like perfect, theoretical, idealized, controlled, etc. to describe the experiment. Consequently, we developed a codebook for the realistic/idealistic code (shown in Table I), and two other researchers (the 2nd and 3rd authors) refined and discussed the the codebook by coding approximately $10 \%$ of student responses. While it is perhaps not surprising that students in the early stages of exposure to computation find the activity to be more idealized than a table-top experiment, the extreme language of "fake" and "ideal" was a surprising finding.

In addition, we found that when students discussed the realistic/idealistic nature of the activities, they also frequently discussed the physics concepts and experimental factors from our coding scheme. This suggests that students are linking particular physics concepts to their assumptions in modeling physical systems. Students also reflected on how the realistic nature of the model is related to controllable factors such as the number of particles (e.g. the ratio of water molecules to beads/solute) and the mass or size of those particles.

\section{B. Affordances and constraints of in-class activities}

The final exam allowed us to analyze students' perceptions of the advantages and disadvantages of computational versus experimental techniques. Specifically, we coded for common affordances and constraints identified by students. The emergent codes from our analysis are shown in Table II.

Similar to the pre/post-lab analysis, the most prevalent affordances and constraints were related to the realistic/idealistic nature of the activities. In the previous section, we noted that many students used terms like "fake" and "perfect" to describe these two activities. In the final exam coding, we are able to unpack these ideas. We find that students perceive the microscope lab as being real, and label that as an affordance of the activity. In a complementary way, students see the computational simulation as ideal, and also label this an affordance. Interestingly, students did not often discuss the lack of "idealness" in the microscope activity as a constraint.

We also find in these responses that students have moved from categorizing the computation/experimentation as merely real or ideal to talking about these activities from two different perspectives: the scientific practices each activity supports and the learning outcomes for diffusion. In the scientific practices, we see responses that are quite sophisticated 
TABLE II. Emergent affordances and constraints (obtained from final exam responses) of the computational modeling activity versus the empirical microscope lab. Affordances and constraints are further categorized as relating to scientific practices or learning outcomes.

\begin{tabular}{|c|c|c|c|}
\hline Code & Sub-code & Computational Activity & Microscope Lab \\
\hline \multirow[b]{2}{*}{ Affordances } & Scientific Practices & $\begin{array}{c}\text { Easily manipulate variables } \\
\text { Teaches physics concepts/theory } \\
\text { Fast, easy, repeatable }\end{array}$ & $\begin{array}{c}\text { Easily test variables } \\
\text { Teaches experimental techniques }\end{array}$ \\
\hline & Learning Outcomes & $\begin{array}{c}\text { Does not include external factors } \\
\text { Microscopic representation } \\
\text { Obtain numerical data } \\
\text { Easy to observe } \\
\text { 3D }\end{array}$ & $\begin{array}{c}\text { Includes external factors } \\
\text { Macroscopic representation } \\
\text { Obtain numerical and visual data } \\
\text { Direct observation, hands-on, interactive }\end{array}$ \\
\hline \multirow{2}{*}{ Constraints } & Scientific Practices & $\begin{array}{l}\text { Programming skill-dependent } \\
\text { Limited by code } \\
\text { Computational power, crashing threshold }\end{array}$ & $\begin{array}{c}\text { Experimentally skill-dependent } \\
\text { Time-consuming, difficult to perform } \\
\text { Expensive equipment } \\
\text { Experimental/human error }\end{array}$ \\
\hline & Learning Outcomes & $\begin{array}{l}\text { Does not include external factors } \\
\text { Difficult to observe }\end{array}$ & $\begin{array}{c}\text { Includes external factors } \\
\text { Difficult to observe or track } \\
\text { Beads settle/slow down quickly } \\
\text { 2D }\end{array}$ \\
\hline
\end{tabular}

with students discussing the ease with which variables can be manipulated and the kind of skills/equipment required to perform these activities. Their responses are not simple characterizations of good versus bad, but rather descriptions of the trade-offs between computational and empirical approaches.

In the learning outcomes, we find that students label particular aspects of both activities as important for studying the topic at hand. These aspects include making easy observations in either 2 or 3 dimensions, being able to account for physical forces in their model, and observations of both microscopic and macroscopic elements of the diffusive process. However, we find that within these categories, the labeling is not universal. For example, students collectively found "including external factors" as an affordance and constraint for both activities.

\section{IMPLICATIONS}

In conclusion, we investigated combining computational modeling with traditional empirical labwork in the classroom. We found that throughout the course of the activities, students described the computational activity as unrealistic and idealized, whereas they described the empirical lab as realistic and accounting for many outside factors. The tension between these ideas is an important opportunity for student learning.
The paired activities supported students in designing experiments that test diffusion in both computational and empirical environments. Our analysis has shown that students expanded their understanding of computational and empirical parameters, and that they better understand how these parameters affect their comprehension of diffusion. We argue that pairing computational simulation with empirical investigation in the context of diffusion supports students in their progress.

In this paper, we have shown that the pairing of these two activities is an opportunity for educators to emphasize the advantages of computational techniques versus experimental techniques. As students are trained to become professionals, recognizing the advantages of differing scientific techniques is a useful skill. Additionally, we propose that students' perceptions of what is real in their science labs can have an impact on their willingness to engage with course material. Thus, by recognizing what makes their computational models and empirical labs more realistic or idealized, students deepen their understanding of the usefulness of one approach over the other.

\section{ACKNOWLEDGMENTS}

We thank the Lyman Briggs College SUTL program at MSU for funding, as well as the Physics Education Research Topical Group for funding our attendance at this conference.
[1] P. Kohl and H. Kuo, Am. J. Phys. 80, 832 (2012).

[2] E. Brewe, Am. J. Phys. 76, 1155 (2008).

[3] J. Kozminski et al., "AAPT Recommendations for the Undergraduate Physics Laboratory Curriculum," AAPT (2014).

[4] B. Geller, B. Dreyfus, J. Gouvea, V. Sawtelle, C. Turpen, and E. Redish, Am. J. Phys. 82, 394 (2014).
[5] S. Tunstall, A. Nair, K. Hinko, P. Irving, and V. Sawtelle, PERC Proceedings, 392 (2017).

[6] K. Moore, J. Giannini, and W. Losert, Am. J. Phys. 82, 387 (2014).

[7] S. Lin, S. Douglas, J. Aiken, C. Liu, E. Greco, B. Thoms, M. Caballero, M. Schartz, PERC Proceedings, 163 (2014). 